\title{
A comparative study of the contrast of solar magnetic elements in $\mathrm{CN}$ and $\mathrm{CH}$
}

\author{
V. Zakharov ${ }^{1}$, A. Gandorfer ${ }^{1}$, S. K. Solanki ${ }^{1}$, and M. Löfdahl ${ }^{2}$ \\ 1 Max-Planck-Institut für Sonnensystemforschung, Max-Planck-Strasse 2, 37191 Katlenburg-Lindau, Germany \\ e-mail: [zakharov; solanki; gandorfer]@linmpi .mpg.de \\ 2 Institute for Solar Physics of the Royal Swedish Academy of Sciences, AlbaNova University Center, 10691 Stockholm, \\ Sweden \\ e-mail: mats@astro.su.se
}

Received 17 March 2005 / Accepted 28 May 2005

\begin{abstract}
Photospheric bright points were investigated in three different wavelength bands using interference filters centered at $436.5 \mathrm{~nm}$ (continuum), $430.5 \mathrm{~nm}$ (Fraunhofer's G-band dominated by absorption due to $\mathrm{CH}$ ), and $388.7 \mathrm{~nm}$ (absorption band of $\mathrm{CN})$. Such bright points serve as proxies of small-scale solar magnetic elements. Near diffraction limited imaging was achieved by real-time frame selection and subsequent joint phase diverse speckle reconstruction.

Comparison of the filtergrams of NOAA0670 taken in $\mathrm{CH}$ and $\mathrm{CN}$ shows that the contrast of bright points is on average 1.4 times higher in $\mathrm{CN}$ than in G-band, which is in good quantitative agreement with the predictions of Berdyugina et al. (2003, A\&A, 412, 513) and Rutten et al. (2001, ASP Conf. Ser., 236, 445).
\end{abstract}

Key words. Sun: activity - Sun: faculae, plages - Sun: magnetic fields

\section{Introduction}

High resolution filtergrams of the solar surface taken in the G-band at around $430.5 \mathrm{~nm}$, dominated by an absorption band of $\mathrm{CH}$, show bright features in the intergranular regions. Although these structures are often elongated along the narrow intergranular lanes, the name intergranular bright points (BP) has been used ever since BPs where first observed in 1984 (Muller \& Roudier 1984). Since then a number of observations (e.g. Berger \& Title 1996; Löfdahl et al. 1998; Langhans et al. 2002; Berger et al. 2004) and theoretical investigations of their radiative properties have been carried out (e.g. Rutten et al. 2001; Carlsson et al. 2004; Keller et al. 2004). Intergranular bright points show a strong spatial correlation with magnetic flux concentrations and are therefore useful as magnetic proxies, which allow the distribution and dynamics of magnetic features to be studied at higher spatial resolution than using spectro-polarimetric techniques. Bright points are also seen on images taken in the blue continuum spectral range, but with significantly less intensity contrast as compared to the G-band.

Recent radiative transfer computations on the basis of realistic MHD simulations indicate that the local brightness excess in $\mathrm{CH}$ filtergrams is due to the higher temperature of magnetic elements at the height of line-core formation of $\mathrm{CH}$ molecular lines as compared to the outside atmosphere (Shelyag et al. 2004). Numerical MHD simulations by Rutten et al. (2001); Steiner et al. (2001) showed that another diatomic molecule in the solar atmosphere, $\mathrm{CN}$, in the region $10^{-4} \leq \tau \leq 10$

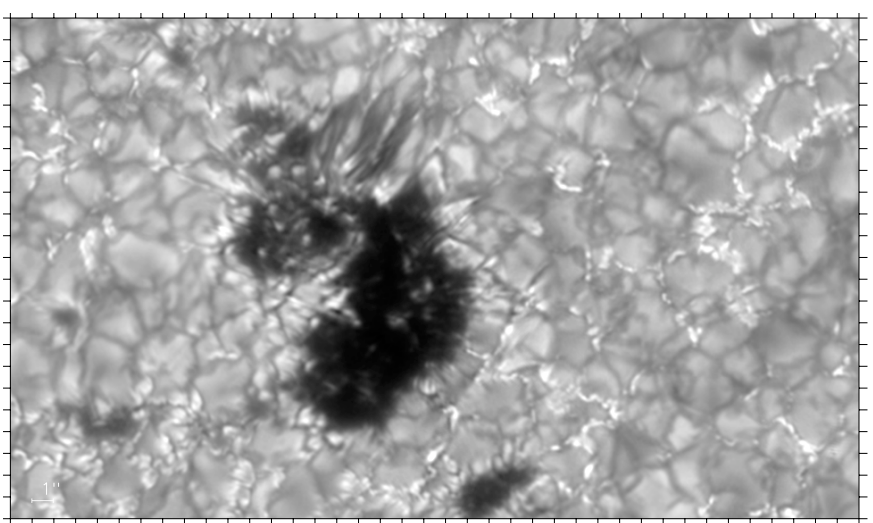

Fig. 1. Joint phase diverse speckle reconstructed $\mathrm{CN}$ band-head image of part of an active region NOAA0669 at $4.91^{\circ} \mathrm{S}, 8.14^{\circ} \mathrm{E}, \mu=0.97$, taken with the SST on 7 September, 2004 at 14:30:15.

behaves very similarly to $\mathrm{CH}$. Computations using plane parallel model atmospheres show that the contrast of a BP is expected to be higher in the UV band-head at $388.3 \mathrm{~nm}$ of $\mathrm{CN}$ than in the G-band (Berdyugina et al. 2003).

The typical size of BPs is around $160 \mathrm{~km}$ (Wiehr et al. 2004), which requires high-resolution observation techniques to spatially resolve them and to provide reliable information on their brightness distribution and structure. Early observations of active regions in the CN band-head (e.g. Chapman 1970; Sheeley 1971) did not possess the necessary spatial resolution. 
In this paper we present a quantitative comparison between the contrast of BPs in the G-band, the $\mathrm{CN}$ band-head, and the blue continuum measured from high resolution filtergrams. We also compare with model predictions.

\section{Observations and analysis}

Observations where carried out at the new Swedish 1-m Solar Telescope (SST) on La Palma in September 2004. We observed different active regions on the Sun containing BPs in three spectral regions. Phase diversity image pairs in the $\mathrm{CN}$ bandhead were recorded on a KODAK Megaplus 4.2 CCD camera (the field of view was reduced to $660 \times 1050$ pixels with a pixel size of $9 \mu \mathrm{m}$ ) through a $0.8 \mathrm{~nm}$ wide interference filter centered at $388.7 \mathrm{~nm}$. Phase diversity image pairs in the Gband $(430.5 \mathrm{~nm})$ and at $436.5 \mathrm{~nm}$ (blue continuum) were captured on two KODAK Megaplus 1.6 CCD $(1536 \times 1024$ pixels with a pixel size of $9 \mu \mathrm{m}$ ) cameras with a $F W H M$ of $1.0 \mathrm{~nm}$. Beamsplitters enabled simultaneous recordings in the three spectral regions. In our observations the exposure time was $12 \mathrm{~ms}$ for $\mathrm{CN}$ and $11 \mathrm{~ms}$ for G-band and continuum. All the filtergrams used here were co-aligned through cross-correlation.

The contrast of small-scale structures in an image is very sensitive to its spatial resolution. Although the theoretical spatial resolution of the SST at $430 \mathrm{~nm}$ is around 0.11 arcsec, purely diffraction limited images are not achieved directly due to wavefront aberrations of two different types: aberrations due to random fluctuations of the refractive index of the earth's atmosphere (seeing) and static aberrations within the telescope itself. In order to achieve nearly diffraction limited images tip-tilt and Adaptive Optics (AO) (Scharmer et al. 2003) corrections with the subsequent joint phase diverse speckle (JPDS) image restoration technique (Paxman et al. 1992; Löfdahl 2002) where used. For our observations the analysis of time sequences of the filtergrams did not show visible motions or structure changes of the BPs up to $130 \mathrm{~km}$ scales in less than $15 \mathrm{~s}$. Therefore, to be conservative, we selected the best 8 phase diversity image pairs obtained within $10 \mathrm{~s}$ for further investigation.

The unbiased comparison of the contrast values of BPs at different wavelengths requires images with equally high spatial resolution in all three spectral regions $(\mathrm{CN}, \mathrm{CH}$ and blue continuum). This is particulary problematic, since the magnitude of the atmospheric aberrations is wavelength dependent in an only approximately and statistically known fashion, but is known to increase towards shorter wavelengths. Although in theory the JPDS algorithm can yield an unaberrated image, due to photometric noise only near-diffraction limited resolution of the restored image can be achieved.

The Phase Diversity algorithm is usually applied to subfields smaller than or on the order of the isoplanatic angle, and a restored frame is made by mosaicking the object estimates from each subfield. For our data, $128 \times 128$ pixel subfields (corresponding to $5.2 \times 5.2$ arcsec) where used. The different wavelengths were restored separately and have different amounts of residual aberrations after the JPDS processing. In order to equalize the spatial resolution of the restored $\mathrm{CN}, \mathrm{CH}$,
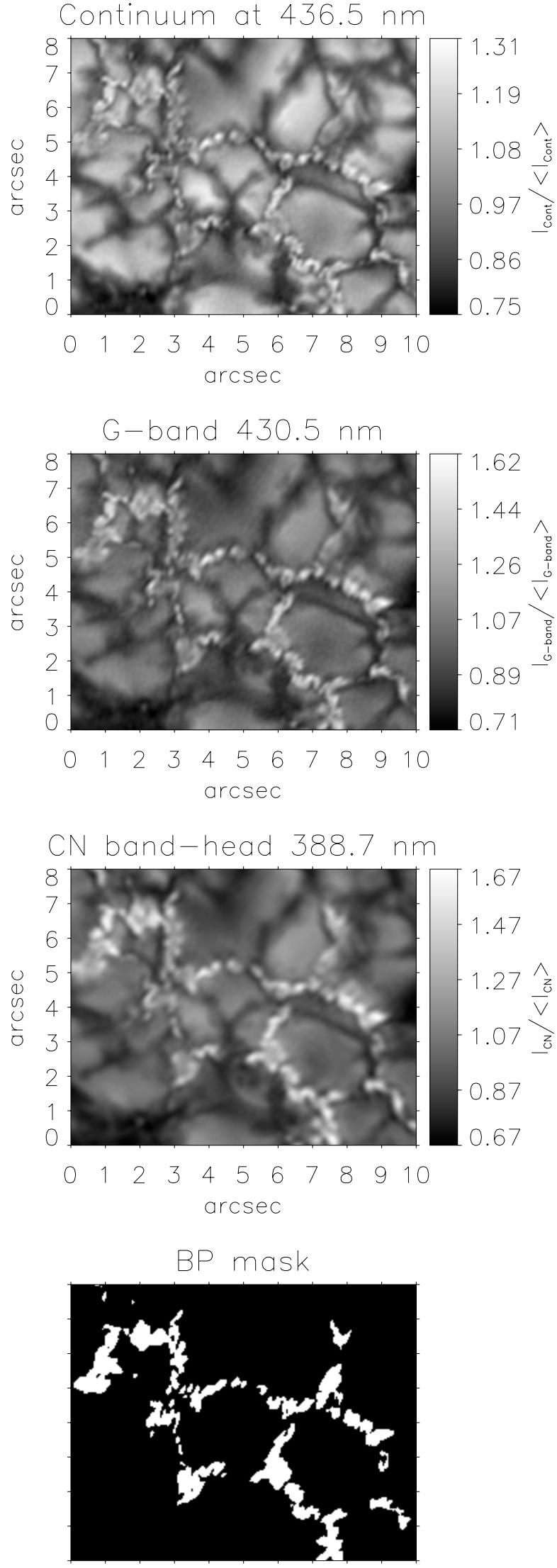

Fig. 2. Joint phase diverse speckle reconstructed filtergrams containig BPs taken in the blue continuum $(436.5 \mathrm{~nm}), \mathrm{G}$-band $(430.5 \mathrm{~nm})$ and $\mathrm{CN}$ band-head (388.7 nm). Also shown (bottom) is the mask used to identify bright points. The plotted images have not been filtered to equalize spatial resolution. 




Fig. 3. Intensity along a cut through a typical BP for the blue continuum (436.5 nm - dashed line), G-band (430.5 nm - dotted line) and $\mathrm{CN}$ (388.7 nm - thick solid line).

and blue continuum images the following technique was applied: in the last step of the JPDS reconstruction, for a given subfield, the final restored $\mathrm{CN}, \mathrm{CH}$ and continuum subfields were filtered with the same spatial filter. This filter was based on the cut-off frequencies, in the Fourier domain, of the worst image, which always was the $\mathrm{CN}$ filtergram, probably due to the lower $\mathrm{S} / \mathrm{N}$ in the original images. This technique works if the sizes of the smallest structures lie below the spatial resolution. Tests using MHD simulations suggest that this is the case.

\section{Results}

In Fig. 2 reduced filtergrams in the blue continuum, G-band and $\mathrm{CN}$ are presented. The contrast for a given pixel assigned to a BP is defined as:

$C_{\lambda}=\frac{I_{\lambda}-I_{0, \lambda}}{I_{0, \lambda}}$,

where $I_{\lambda}$ denotes the intensity of the BP in a certain wavelength band $\lambda$ and $I_{0, \lambda}$ is the mean intensity of the quiet sun for the same wavelength. Intensities of BP pixels vary over a wide range. Often BPs are darker than granules (Langhans et al. 2002; Shelyag et al. 2004). In order to allow a secure and automated identification of BPs we use the technique of Berger et al. (1998), which consists of the substraction of the continuum image from the corresponding G-band image.

In the bottom image in Fig. 2 the binary mask used to identify regions corresponding to all BPs, which according to Shelyag et al. (2004) correspond to a mean temperature at the layer of the continuum intensity formation $\left\langle T_{\tau \approx 2 / 3}\right\rangle$ of $\approx 6500 \mathrm{~K}$ is shown. The three reconstructed filtergrams were co-aligned, so that the same BP-mask could be used. Figure 3 shows the intensity variations along the same cut in a typical BP in the three spectral regions. The intensities are normalized to the average quiet Sun values. The calculated mean contrast values of the BPs are presented in Table 1. The contrast of the BPs is higher in $\mathrm{CN}$ than in the G-band, with the mean ratio $\left\langle C_{\mathrm{CN}} / C_{\mathrm{CH}}\right\rangle=1.4$ over all BPs in the $27 \times 43$ arcsec filtergram triplet.
Table 1. Observed contrast values of BPs with respect to the quiet Sun intensity within the G-band at $430.5 \mathrm{~nm}$, the violet $\mathrm{CN}$ bandhead at $388.7 \mathrm{~nm}$ and blue continuum at $436.5 \mathrm{~nm} .\langle C\rangle,\left\langle C^{\mathrm{JPDS}}\right\rangle$ and $\left\langle C^{\mathrm{JPDS}+\text { equ }}\right\rangle$ denote the contrast in the not reconstructed, JPDS reconstructed and JPDS reconstructed with resolution equalization images respectively. rms contrasts are computed for the quiet Sun.

\begin{tabular}{lllll}
\hline \hline Spectral region & $\langle C\rangle$ & $\left\langle C^{\mathrm{JPDS}}\right\rangle$ & $\left\langle C^{\mathrm{JPDS}+\text { equ }}\right\rangle$ & $\mathrm{rms}$ \\
\hline Continuum & 0.090 & 0.145 & 0.143 & 0.11 \\
G-band & 0.218 & 0.341 & 0.340 & 0.12 \\
$\mathrm{CN}$ & 0.311 & 0.481 & 0.481 & 0.12 \\
\hline
\end{tabular}

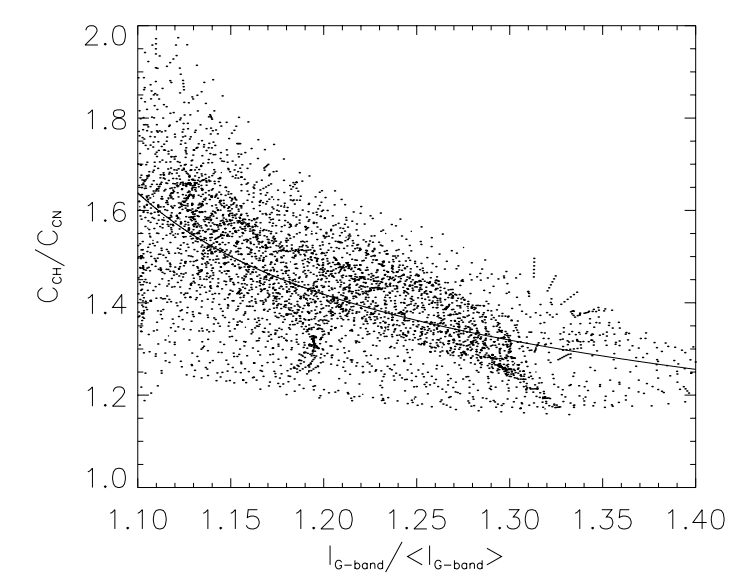

Fig. 4. Scatter plots of the contrast ratio in CN band-head and G-band vs. normalized G-band intensity. The solid line represents a 4th degree polynomial least squares fit to the data points.

A statistical study of the brightness distributions of BPs in $\mathrm{CN}$ and $\mathrm{CH}$ filtergrams covering $81 \times 43$ arcsec of active regions with the filling factor $\alpha \approx 0.03$ showed that the contrast ratio of the BPs in $\mathrm{CN}$ to $\mathrm{CH}$ decreases with increasing G-band brightness of the BPs (Fig. 4). In Fig. 4 only a subset of the analyzed data is shown for a subfield, for which the alignment of the various images was particulary good.

\section{Conclusions}

Our analysis of filtergrams of an active region in the G-band, the $\mathrm{CN}$ band-head and the blue continuum shows that the mean contrast value of inter-granular bright points with respect to the quiet Sun in $\mathrm{CN}$ is typically $C_{\mathrm{CN}} / C_{\mathrm{CH}} \approx 1.4$ times higher than in G-band and $C_{\mathrm{CN}} / C_{\text {cont }} \approx 3.4$ times higher than in the blue continuum, which is in reasonable agreement with simple theoretical predictions: $C_{\mathrm{CN}}^{\text {theor }} / C_{\mathrm{CH}}^{\text {theor }}=1.4, C_{\mathrm{CN}}^{\text {theor }} / C_{\mathrm{cont}}^{\text {theor }}=2.8$ (Berdyugina et al. 2003; Kiselman et al. 2001). The contrast of BPs in the G-band is $C_{\mathrm{CH}} / C_{\text {cont }} \approx 2.4$ times higher than in the blue continuum, also in rough agreement with their predictions: $C_{\mathrm{CH}}^{\text {theor }} / C_{\text {cont }}^{\text {theor }}=2.0$.

Theoretically the relative brightness excess of BPs in $\mathrm{CN}$ with respect to G-band is due to a stronger decrease of the number density of the $\mathrm{CN}$ molecule with increasing temperature at the level of line-coreformation, as compared to $\mathrm{CH}$. Although 
the observed contrast ratios agree reasonably with model predictions, the absolute contrast values of observed BPs are lower than those in theoretical predictions based on the Kurucz model atmosphere (Kurucz 1993) with $T_{\tau \approx 2 / 3} \approx 6500 \mathrm{~K}$ by a factor of 5 .

The difference between the absolute contrasts found from the observations and computed by Berdyugina et al. (2003) can be attributed to several factors, the main ones of which are: 1) The computed contrasts where calculated using a simple Kurucz model whose temperature stratification along $\tau$ differs from that of a flux-tube in the photosphere even if the temperatures at $\tau \approx 2 / 3$ are equal; 2 ) the computed contrasts refer to an unlimited spatial resolution whereas our observed filtergrams are limited to a spatial resolution of $130 \mathrm{~km}$; 3) the observed contrast is further diminished by scattered light. Alternatively, the temperature contrast between magnetic BPs and the quiet Sun is lower.

The significantly higher contrast seen in the violet $\mathrm{CN}$ band-head compared with the G-band makes it a promising wavelength band for solar high resolution studies, although there are factors making contemporary high-resolution groundbased solar observations in this wavelength more complex than in the G-band: 1) fewer photons are provided by the Sun; 2) there is more scattered light; 3 ) the spatial resolution is worse because of the stronger influence of the atmospheric aberrations (seeing).

A detailed synthesis of this wavelength band using 3D MHD simulations, as well as simultaneous high resolution spectra of $\mathrm{CN}$ and $\mathrm{CH}$ lines would also be of considerable interest.

Acknowledgements. We are grateful to G. Scharmer for help with the instrumentation, S. Berdyugina for helpful discussions \& R. Rutten for carefully reading and improving the manuscript. The SST is operated by the Royal Swedish Academy of Sciences at the Spanish Observatorio del Roque de los Muchachos of the Instituto de Astrofisica de Canarias.

\section{References}

Berdyugina, S. V., Solanki, S. K., \& Frutiger, C. 2003, A\&A, 412, 513 Berger, T. E., \& Title, A. M. 1996, ApJ, 463, 365

Berger, T. E., Löfdahl, M. G., Title, A. M., \& Shine, R. S. 1998, ApJ, 495, 973

Berger, T. E., Rouppe van der Voort, L. H. M., Löfdahl, M. G., et al. 2004, A\&A, 428, 613

Carlsson, M., Stein, R., Nordlund, A., \& Sharmer, G. 2004, IAU Symp., 223, Multi-Wavelength Investigations of Solar Activity, ed. A. V. Stepanov, E. E. Benevolenskaya, \& A. G. Kosovichev, 233

Chapman, G. A. 1970, Sol. Phys., 18, 78

Keller, C. U., Schüssler, M., Vögler, A., \& Zakharov, V. 2004, ApJ, 607, 59

Kiselman, D., Rutten, R. J., \& Plez, B. 2001, Recent Insights into the Physics of the Sun and Heliosphere, ed. P. Brekke, B. Fleck, \& J. B. Gurman, ASP Conf. Ser., 200, 287

Kurucz, R. L. 1993, Vizier On-line Data Catalog, VI/39

Langhans, K., Schmidt, W., \& Tritschler, A. 2002, A\&A, 394, 1069

Löfdahl, M. G. 2002, in Image Reconstruction from Incomplete Data II, ed. P. J. Bones, M. A. Fiddy, \& R. P. Millane, Proc. SPIE, 4792, 146

Löfdahl, M. G., Berger, T. E., Shine, R. S., \& Title, A. M. 1998, ApJ, 495, 965

Muller, R., \& Roudier, T. 1984, Sol. Phys., 94, 33

Paxman, R. G., Schulz, T. J., \& Fienup, J. R. 1992, Opt. Soc. America, 9, 1072

Rutten, R. J., Kiselman, D., Rouppe van der Voort, L., \& Plez, B. 2001, Advanced Solar Polarimetry - Theory, Observation, and Instrumentation, ed. M. Sigwarth, ASP Conf. Ser., 236, 445

Scharmer, G. B., Dettori, P. M., Peter, M., Löfdahl, M. G., \& Shand, M. 2003, in Innovative Telescopes and Instrumentation for Solar Astrophysics, ed. S. L. Keil, \& S. V. Avakyan, Proc. SPIE, 4853, 370

Sheeley, N. R. 1971, Sol. Phys., 20, 19

Shelyag, S., Schüssler, M., Solanki, S. K., Berdyugina, S. V., \& Vögler, A. 2004, A\&A, 427, 335

Steiner, O., Hauschildt, P., \& Bruls, J. 2001, A\&A, 372, 13

Steiner, O., Hauschildt, P., \& Bruls, J. 2003, Astron. Nachr., 324, 398

Wiehr, E., Bovelet, B., \& Hirzberger, J. 2004, A\&A, 422, 63 